\title{
Comparison of the characteristics of the genetic algorithm and the method of coordinates search for optimization of temperature modes indoor areas
}

\author{
A P Shuravin ${ }^{1}$, S V Vologdin ${ }^{1}$ \\ ${ }^{1}$ Kalashnikov Izhevsk State Technical University, Studencheskaya, 7, Izhevsk, Russia, \\ 426069 \\ e-mail: webmaster@easyprog.ru,vologdin_sv@mail.ru
}

\begin{abstract}
The article substantiates the relevance of optimization algorithms research for solving various applied problems and for the science of artificial intelligence. The need to solve problems of optimizing the thermal-hydraulic modes of buildings (as part of the project "Smart City") is explained. The paper presents a mathematical formulation of the problem of optimizing the temperature mode of rooms using adjustable devices. Existing work provides two methods for solving the posed problem. They are the coordinates search method and the genetic algorithm. The article contains the description of the above mentioned algorithms (including the mathematical apparatus used). The results of the computational experiment (for the considered optimization methods) are presented. These experimental results show that the genetic algorithm provides better optimization results than the coordinates search method, but it has a large computational cost. The hypothesis was confirmed that in order to increase the efficiency of solving the considered class of problems it is necessary to combine the genetic algorithm and the coordinates search method.
\end{abstract}

\section{Introduction}

Currently, work in the field of development and implementation of energy efficient technologies is very relevant. The main provisions defining the need for work to improve the energy efficiency of the heating system of buildings are enshrined in Federal Law No 261-F3 “On Energy Saving ...”, as well as by the decree of the Government of the Russian Federation of 13.11.09 Number 1234-p "Energy Strategy of Russia for the period until 2020". In particular, the system solution for optimization of central heating systems at different levels of the organization (district, city, individual buildings) is considered in work[1], as well as the information system IASTS, designed for automated calculation of heat networks and storing information about calculated network is presented. Energy efficiency issues are raised in the program "Smart City" [2]. The study of the joint operation of two sources of thermal energy to optimize the operation of the heating network is considered in [3]. Mathematical model of the building thermal conditions (with consideration for insolational heat input) is described in [4]. In work [5] the "Smart Home" technology is considered as the energy-saving technology of the future. The authors come to the conclusion that, at present, 
the "Smart Home" technology is not widely spread in Russia, but is gradually gaining popularity. The impact of energy-saving technologies on the economy and the future is discussed in [6, 7], new advances in energy saving are discussed in [8]. The connection between energy-saving policy and environment are considered in [9]. The article discusses creating energy system with activeadaptive network in Russia.

One of the methods of energy saving is the optimization of energy consumption including the use of artificial intelligence technologies [10,11]. One of the important areas of research in the field of energy saving is the optimization of the thermo-hydraulic modes of centralized heating systems [1]. Issues of the study of problems and methods for optimizing thermal-hydraulic modes in multicircuit thermal networks and other problems of energy saving are considered in the works of many Russian [1, 10-18] and foreign [19-22] authors.

In particular, in [10] a review of optimization problems of central heating networks is made and the need to automate the solution of these problems is justified. It is proposed to use a genetic algorithm to optimize the thermo-hydraulic modes of buildings in [11]. Multi-criteria optimization problems are considered in [12]. As a rule these problems arise before the beginning of the heating season. In [13] a mathematical model is presented for optimizing the thermal power of heat sources operating in the centralized heat supply system. A new method for solving the problem is considered, the results of test problems calculations are presented. A mathematical model for optimizing heat flows between subscribers of the heating network in order to reduce the disbalance of the heating system, including the methodology for solving such problems, is considered in [14]. There is a problem of operative regulation of heat supply depending on the outdoor temperature. This problem is solved in [15] by applying simulation. A method for optimizing the temperature of the coolant based on the use of penalty functions for indoor air temperature and the amount of heat energy consumed is considered. In [16] an issue of optimal distribution of reactive power in power supply systems and electrical networks is investigated. For these purposes, the authors proposed to apply the algorithm of swarm intelligence. In [17] a method of thermal network equivalence allows replacing a real network with a mathematical model is considered. The method is used to optimize of heating system. The ideas and dependencies that served as the basis for the creation of this model are given in the article. The adequacy of the equivalent model is substantiated. The advantages of thermal network equivalence in terms of simplifying the calculation of various modes are noted. Complex problem of schematic structural optimization of heat networks is considered in [18]. For solving this problem authors used tree search algorithm and annealing simulation method.

There are a lot of foreign papers about energy system optimization. The article [19] shows Monte Carlo method application for energy system optimization, modelling of safety, energy transfer failures at real time. The paper [20] shows optimization problems solving in electricenergy industry to decrease cost with consideration of safety. In work [21] a hierarchical optimization model for a network of charging stations for electric vehicles is considered. The authors proposed a three-layer optimization model, including optimization of gas station locations (first layer), queuing model (second layer), and optimization of battery charging process (third layer). A kriging model to reduce energy losses in distribution networks is proposed in [22]. The authors presented an algorithm for the approximation of complex distribution networks to speed up the process of finding a solution.

The development of new optimization algorithms and the study of already existing algorithms are relevant [23-25]. For example, the decentralized training algorithm is described in [23]. It is used in distributed big data application. The Constant Jacobian Gauss-Newton Optimization Algorithm is presented in [24]. Work [25] describes optimal cost almost-sure reachability in POMDPs.

Each of the optimization algorithms has certain disadvantages, for example, the lack of guarantees for achieving the global extremum of the objective function, high computational cost, etc. $[26,27]$. 
Based on the foregoing, the purpose of this work is an experimental study and comparative analysis of various approaches to optimizing the thermal-hydraulic modes of buildings. This work is supported by grant 27.06.01/18BCB of Kalashnikov ISTU.

\section{The problem of optimizing the temperature of the indoor areas}

Practice shows that in order to make optimal management decisions it is necessary to solve the problems of analyzing the thermo-hydraulic mode and characteristics optimization at different levels of HSS in complex with using mathematical algorithms of decreasing heat supply system disbalance, energy audit methods and computer methods of data processing for visualize and analyze heat network any elements (table 1).

Table 1. Methods of increasing energy efficiency HSS.

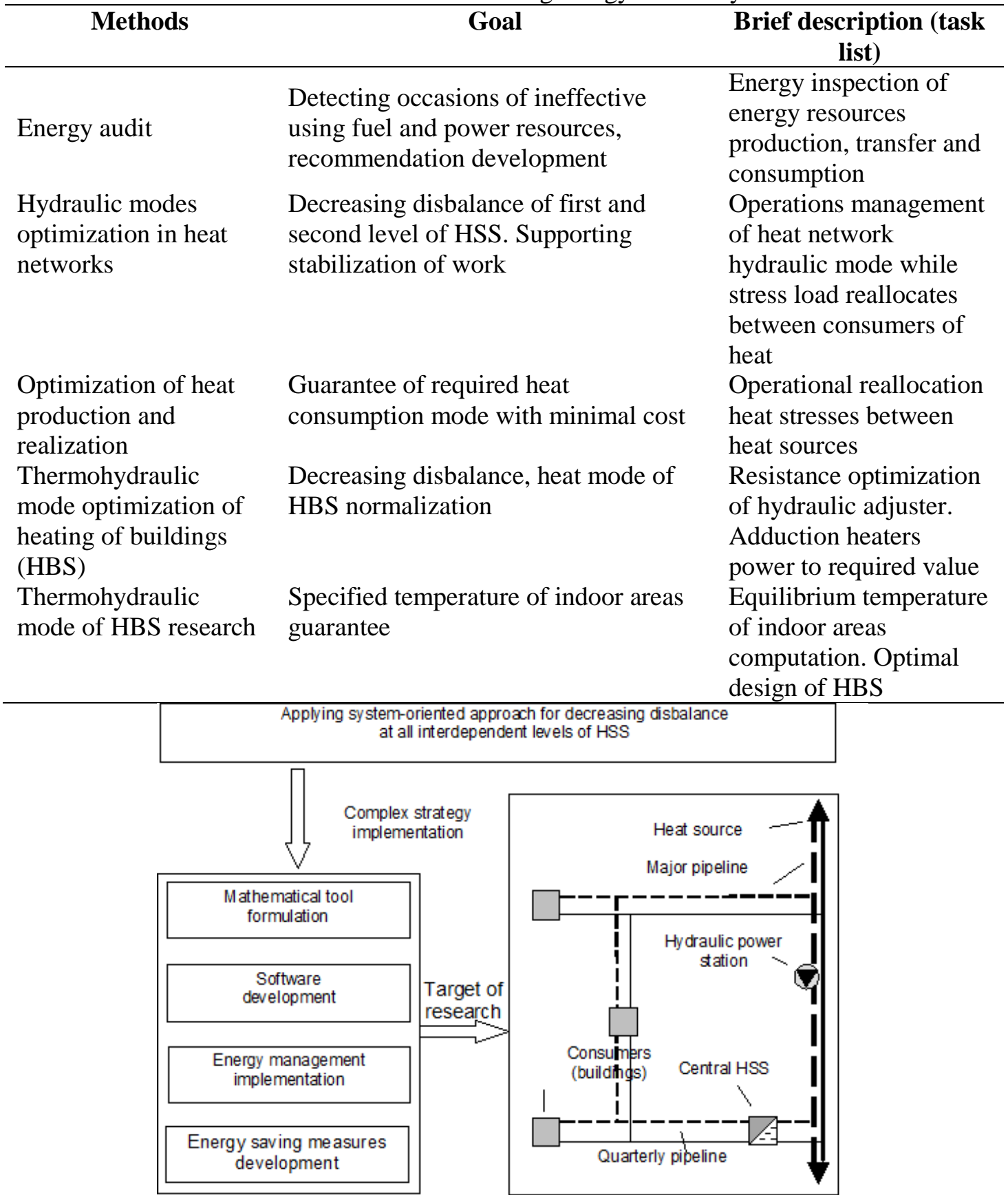

Figure 1. System-based approach applied to decreasing disbalance of central HSS (solid - cold source, dashed - hot source). 
Principal elements and strategy of system-oriented approach for increasing heat efficiency of HSS through a disbalance reduction are showed at figure. 1.

Mathematical model construction methodology of decreasing disbalance of central HSS based on applying characteristics optimization methods at different levels of HSS is presented at figure 2. Methodology comprises methods: power optimization of heat sources (first level HSS), heat flows optimization between heat network subscribers (second level), resistance optimization of heater system hydraulic adjusters (third level).

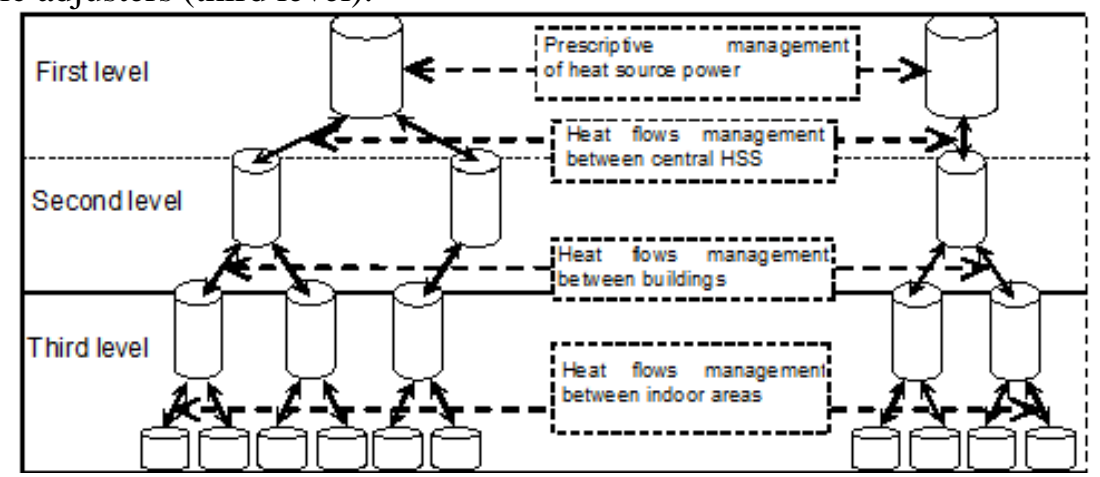

Figure 2. Generalized chart of heat flows management in centralized HSS

In this study the energy system is optimized at the third level (heat flows management between indoor area).

Excessive energy consumption of a building heat supply system arises for the following reasons:

- ineffective thermal protection of the indoor areas;

- non-optimal scheme of heat supply of the building;

- due to the temperature disbalance of the indoor areas of heated buildings (to raise the temperature of the "cold" rooms up to the standard, it is necessary to increase the coolant temperature, while in the rooms where the temperature is normal, it becomes too hot, and consumers have to open the windows "heating the outdoor").

There are several ways to reduce the temperature disbalance of the indoor areas:

- by selection of the optimal power of the heating devices of the building indoor areas, ensuring the standard temperature in each room, taking into account the actual state of the enclosing structures (as a rule, this approach is carried out at the stage of building design or reconstruction of buildings);

- by regulating the balancing valves of the risers, this issue is considered in [11] (it is applicable to existing and projected buildings);

- by regulating the adjusting valves of the heaters (applicable to existing buildings), this issue is addressed in this article.

It is worth noting that by adjusting the balancing valves of the risers it is impossible to eliminate the disbalance of those rooms that are connected to the same riser. The working hypothesis of the study carried out under this article is that regulating the valves of the heaters, as well as the joint regulation of the valves of the heaters and the balancing valves of the risers should give the best optimization results.

The objective function of the problem of optimizing the temperature disbalance is the minimum of the standard deviation of the room temperature from the standard value:

$$
\Phi\left(S_{1}, S_{2}, \ldots, S_{z}\right)=\left[\frac{1}{N} \sum_{i=1}^{N}\left(t_{i n, i}\left(S_{1}, S_{2}, \ldots, S_{z}\right)-t_{o p t}\right)^{2}\right]^{1 / 2} \rightarrow \min ,
$$

where $z$ is the quantity of regulating valves of the heating devices and balancing valves of the risers, $i$ is the number of indoor area, $N$ is the quantity of indoor areas, $t_{i n, i}$ is the design room temperature (the method of calculation it will be mentioned below), $t_{o p t}$ is the indoor area 
temperature, which is planned to be reached (in the article, two optimization options are applied: relative to the standard temperature and the average temperature of the rooms in the building, will be considered below), $S_{j}$ is the $j$-valve resistance.

Due to the fact that the control valves have their own range of resistance values, we introduce a restriction:

$$
S_{j}^{\min } \leq S_{j} \leq S_{j}^{\max },
$$

where $S_{j}^{\min }$ and $S_{j}^{\max }$ are minimum and maximum resistance respectively [11].

The air temperature inside the heated indoor areas $\left(t_{i n, i}\right)$ is calculated from the heat balance equation [1], which takes into account the heat influx from the coolant in heating devices and heat losses through the enclosing structures (walls, windows, doors, floor, etc.):

$$
Q_{w l}^{H}+Q_{f l}^{H}+Q_{c l}^{H}+Q_{d r}^{H}+Q_{d r}^{I}+Q_{w i n}^{H}+Q_{w i n}^{I}+Q_{a i r}^{I}=Q_{r d}+Q_{p},
$$

where $Q_{w l}^{H}$ is heat exchange with walls, $Q_{f l}^{H}$ is heat exchange with a floor, $Q_{c l}^{H}$ is heat exchange with a cell, $Q_{d r}^{H}$ is heat exchange with doors, $Q_{d r}^{I}$ is infiltration through doors, $Q_{w i n}^{H}$ is heat exchange with windows, $Q_{w i n}^{I}$ is infiltration through windows, $Q_{a i r}^{I}$ is infiltration of air, $Q_{r d}$ is heat influx from radiators, $Q_{p}$ is heat influx from pipes.

The equation (3) is written and solved separately for each room from the $N$ rooms of the building. The equation is solved with iteration method at which the temperature is selected corresponding to the temperature equilibrium.

Heat transfer through the walls (similarly floor, ceiling, window and balcony structures) depends on the area of the walls, their material, thickness and temperature difference:

$$
Q_{w l}^{H}=\frac{F_{w l}}{R_{w l}}\left(t_{\text {in }}-t_{\text {out }}\right) n \beta,
$$

where $F_{w l}$ is area of walls, $R_{w l}$ is reformed wall heat transfer resistance; $t_{i n}$ is indoor air temperature (in formulae (3) it is $\left.t_{i n, i}\right)$; $t_{\text {out }}$ is outdoor temperature; $n$-tabular correction factor depending on the type of fencing surface; $\beta$ is correction factor taking into account the orientation of the wall relative to the cardinal points.

Heat losses due to the heating of the outside air inflowed through the windows (similar to the entrance doors) are calculated by the formula:

$$
Q_{\text {win }}^{I}=A_{\text {win }}^{I} G_{\text {win }}^{I} F_{\text {win }} c\left(t_{\text {in }}-t_{\text {out }}\right),
$$

where $A_{\text {win }}^{I}$ is correction factor, taking into account the kind of window; $F_{\text {win }}$ is estimated window area; $G_{w i n}^{I}$ is the volume of infiltrated air; $c$ is the specific mass density of air.

Heat transfer is determined on the basis of its heat flow per unit area. Heat flow depends on water flow:

$$
q_{r d}=q_{\text {nom }}\left(\frac{\Delta t_{t p}}{70}\right)^{n+1}\left(\frac{x_{r d}}{360}\right)^{p} c_{r d},
$$

where $x_{r d}$ is actual water consumption in the device, $\mathrm{kg} / \mathrm{h} ; n, p$ are empirical coefficients, $c_{r d}$ is coefficient taking into account the scheme of connection of the heating device; $\Delta t_{t p}$ is temperature pressure equal to the difference between the half sum of the input and output temperature and the air temperature in the room, $q_{\text {nom }}$ is nominal heat flux density.

To determine coolant flow rates, the system of hydraulic equations is solved:

$$
\left\{\begin{array}{c}
A x=q_{\partial}, \\
B y=0, \\
y+h_{\partial}=S X x,
\end{array}\right.
$$

where $A$ is the junction matrix of ramification and node, $B$ is the contour matrix built on the original graph, $x$ and $y$ are vectors of unknown variables, $S$ is the hydraulic resistance matrix 
(include resistance heaters and pipes), $X$ is the consumption matrix, $h_{\partial}$ is the hydraulic pressures vector, $q_{\partial}$ is the vector of coolant consumption in nodes.

The heater having valve connected in parallel with the cross-connection, the hydraulic resistance of such a system is expressed by the formula [1]:

$$
S_{\text {res }}=\frac{1}{\left[\left(\sqrt{S_{r d}}\right)^{-1}+\left(\sqrt{S_{j m}}\right)^{-1}\right]^{2}} .
$$

where $S_{r d}$ is the hydraulic resistance of the heater, $S_{j m}$ is the hydraulic resistance of the jumper. The resistance of the heater is the sum of the resistance of the radiator and the adjusting valve:

$$
S_{r d}=S_{\text {rad }}+S_{\text {valv }} \text {. }
$$

where $S_{\text {rad }}$ is it's own resistance of the heater, $S_{\text {valv }}$ is the resistance of the heater valve.

During the optimization it is necessary to obtain the temperature of the indoor area $\left(t_{i n, i}\right)$ as close as possible to the desired $\left(t_{\text {opt }}\right)$. There are two options to define the temperature:

- standard temperature, according to GOST $30494-2011$ is equal to $20^{\circ}$ [28];

- average temperature. It is calculated by formulae:

$$
t_{\text {opt }}=\frac{\sum_{i}^{N} t_{i n, i}}{N} .
$$

\section{Description of the algorithms used}

Indoor area temperature cannot be analytically expressed from functional (1), therefore, only some iterative optimization methods can be used to find it. Since the functional is non-linear, we exclude methods of linear programming, such as, for example, the simplex method, from the considered methods. It is also impossible to analytically express $t_{\text {in }}$ from equation (3), formulas (4-6) and system of equations (7), thus, it is also calculated by an iterative method. Therefore, it is also impossible to find the derivative of the functional (1), which excludes the possibility of applying gradient methods that require calculating the derivative, such as, for example, gradient descent methods. Based on these limitations, for the purposes of the study, the method of coordinate search and the genetic algorithm are chosen.

In general, the algorithm for optimizing the temperature disbalance of buildings works as follows [11]:

1. The initial values of the resistance of the valves of the raisers and the heating devices are set. Installation method is random value from the admissible range.

2. The structure of the heating network and characteristics of the indoor areas, including enclosing structures is read from the database.

3. The thermo-hydraulic balance of the heating system is calculated.

4. The air temperature in the indoor areas by calculating the equilibrium temperature of the building is calculated [1].

5 . The objective function is calculated.

6. If the stop condition is reached (in this study it is the specified number of iterations), then program exits the loop.

7. The values of the valves resistance of the risers and the heaters are selected. The selection method depends on the optimization algorithm (more will be described below).

8. The program goes to step 2 .

In computer science a genetic algorithm (GA) is a metaheuristic inspired by the process of natural selection that belongs to the larger class of evolutionary algorithms (EA). This is a heuristic search algorithm used to solve optimization and modeling problems by randomly selecting, combining, and varying the desired parameters using mechanisms similar to natural selection in nature. The problem is formalized in such a way that its solution can be encoded as a vector ("genotype") of genes, where each gene can be a bit, a number, or some other object. In some ways (usually random) many genotypes of the initial population are created. They are evaluated using the 
objective function, as a result of which a certain value is associated with each genotype which determines how well the phenotype described by it solves the set problem. From the resulting set of solutions ("generations"), taking into account the value of objective function, solutions are selected (usually the best species are more likely to be selected), to which "genetic operators" are applied (in most crossover and mutation), resulting in new solutions. For them, the value of objective function is also calculated, and then the selection of the best decisions in the next generation is made. This set of actions is repeated iteratively, so the "evolutionary process" is modeled, which lasts several life cycles (generations) until the criterion for stopping the algorithm is fulfilled.

When using the genetic algorithm, selection performs by random mutations (random changes according to the formula) [29]:

$$
\Delta S_{j}=\sigma \sqrt{-2 \ln \left(x_{1}\right)} \cdot \cos \left(2 \pi x_{2}\right),
$$

where $\sigma$ is the mutation step (selected empirically), $x_{1}$ and $x_{2}$ are random values in limits [0;1], having a uniform distribution (such random number generators are embedded in many programming languages). Due to formulae (11) we obtain Gaussian distribution from uniform distribution.

Also sets of valve resistance values can be crossed (mixed randomly) [11]. The probability of crossing is calculated by the formula:

$$
p_{\text {cross }}=\frac{2 n-k_{1}-k_{2}}{2 n-1},
$$

where $n$ is the quantity of sets in the sample («species» in the "population»), $k_{1}$ and $k_{2}$ are respectively, the number of the first and the second set of a pair, which is checked for the possibility of crossing. They take values from 0 to $n-1$. The removal of sets from the sample during selection also occurs randomly; the probability of deletion is the greater, the worse the objective function. This probability is expressed by the formula:

$$
p_{\text {del }}=\frac{m}{n},
$$

where $m$ is the number of the set to be deleted (starting from zero). For the set with the best objective function this number is 0 . It means that this species will never be deleted. The deletion cycle can be repeated several times until the number of sets is less than the maximum allowable number. If the number of the set becomes less than the minimum allowable amount then at this iteration selection will not be made.

In the coordinates search method, the value of valve resistance at the next iteration is changed by increment $\Delta S$, calculated by the formula [1]:

$$
\Delta S_{j}^{(k)}=\frac{\left(s_{j}^{(k)}-s_{j}^{(k-1)}\right)\left(t_{o p t, j}-t_{i n_{j}}^{(k)}\right)}{\left(t_{i n_{j}}^{(k)}-t_{i n_{j}}^{(k-1)}\right)},
$$

where $j=1, \ldots, z$ is the valve number, $k$ is the iteration number, $t_{o p t, j}$ is the required room temperature, $t_{i n_{j}}^{(k)}$ is the indoor area temperature according to $j$-valve (room where set radiator with the valve or room according to ramification which adjusting by the riser valve) on the $k$-th iteration.

\section{Experimental results}

In this study, in order to compare the minima of achieving the objective function, the following experiments were conducted:

- optimization of the hydraulic resistance of the valves of the heating devices;

- optimization of the hydraulic resistances of the balancing valves of the heating risers;

- joint optimization of the hydraulic resistance of the valves of the heating devices and the balancing valves of risers.

For optimization the genetic algorithm and the coordinates search algorithm were used. In the genetic algorithm the mutation step $\sigma$ [11] was taken 5 and 20 in units of $\mathrm{Pa} /(\mathrm{Kg} / \mathrm{h})^{2}$. A typical building was calculated under the average designed conditions of Izhevsk for the actual condition of enclosing structures and space heaters. Optimization was performed in two versions: 
- relative to the standard temperature, which was adopted by $20^{\circ} \mathrm{C}$;

- relative to the average temperature in the rooms.

The initial approximation is a random value from the range of formula (2). Comparison of results is given in tables $2-5$.

Table 2. Comparison of optimization results (values of the objective function relative to the standard temperature $20^{\circ}$ ).

\begin{tabular}{lccc}
\hline & \multicolumn{3}{c}{ Rtandard temperature 20 $).$} \\
& Regulation of & $\begin{array}{c}\text { Regulation of } \\
\text { battery valves } \\
\text { only }\end{array}$ & $\begin{array}{c}\text { Regulation } \\
\text { battery valves and } \\
\text { riser valves }\end{array}$ \\
\hline $\begin{array}{l}\text { Coordinates search } \\
\text { method }\end{array}$ & 3.46 & 3.25 & 2.74 \\
$\begin{array}{l}\text { Genetic algorithm with } \\
\text { step 5 }\end{array}$ & 3.08 & 3.68 & 3.51 \\
$\begin{array}{l}\text { Genetic algorithm with } \\
\text { step 20 }\end{array}$ & 2.85 & 3.68 & 3.40 \\
$\begin{array}{l}\text { No adjustment (all } \\
\text { valves open) }\end{array}$ & 5.14 & 5.14 & 5.14 \\
\hline
\end{tabular}

Table 3. Comparison of optimization results (values of the objective function relative to the average temperature).

\begin{tabular}{lccc}
\hline & \multicolumn{2}{c}{ Regulation of } \\
riser valves only & $\begin{array}{c}\text { Regulation of } \\
\text { battery valves } \\
\text { only }\end{array}$ & $\begin{array}{c}\text { Regulation } \\
\text { battery valves and } \\
\text { riser valves }\end{array}$ \\
\hline $\begin{array}{l}\text { Coordinates search } \\
\text { method }\end{array}$ & 3.21 & 2.91 & 2.67 \\
$\begin{array}{l}\text { Genetic algorithm with } \\
\text { step 5 }\end{array}$ & 2.85 & 2.94 & 2.82 \\
$\begin{array}{l}\text { Genetic algorithm with } \\
\text { step 20 }\end{array}$ & 3.15 & 2.94 & 3.1 \\
$\begin{array}{l}\text { No adjustment (all } \\
\text { valves open) }\end{array}$ & 3.26 & 3.26 & 3.26 \\
\hline
\end{tabular}

Table 4. Optimization by a genetic algorithm after optimization by coordinates search (values of the objective function relative to the standard temperature $\left.20^{\circ}\right)$ ).

\begin{tabular}{lccc}
\hline & $\begin{array}{c}\text { Regulation of } \\
\text { riser valves only }\end{array}$ & $\begin{array}{c}\text { Regulation of } \\
\text { battery valves } \\
\text { only }\end{array}$ & $\begin{array}{c}\text { Regulation } \\
\text { battery valves and } \\
\text { riser valves }\end{array}$ \\
\hline $\begin{array}{l}\text { Coordinates search } \\
\text { method }\end{array}$ & 3.21 & 3.25 & 2.74 \\
$\begin{array}{l}\text { Genetic algorithm with } \\
\text { step 20 }\end{array}$ & 2.95 & 2.99 & 1.96 \\
\hline
\end{tabular}

Table 5. Optimization by a genetic algorithm after optimization by coordinates search (values of the objective function relative to the average temperature).

\begin{tabular}{lccc}
\hline & $\begin{array}{c}\text { Regulation of } \\
\text { riser valves only }\end{array}$ & $\begin{array}{c}\text { Regulation of } \\
\text { battery valves } \\
\text { only }\end{array}$ & $\begin{array}{c}\text { Regulation } \\
\text { battery valves and } \\
\text { riser valves }\end{array}$ \\
\hline $\begin{array}{l}\text { coordinate search } \\
\text { method }\end{array}$ & 3.21 & 2.91 & 2.67 \\
$\begin{array}{l}\text { Genetic algorithm } \\
\text { with step 20 }\end{array}$ & 2.93 & 2.6 & 1.94 \\
\hline
\end{tabular}


As can be seen from Tables 1 and 2, optimization by regulating the valves of the heating devices exceeded optimization by regulating the valves of the risers only in the case of using the coordinates search. However, if after optimization by the coordinates search method, the valve resistance is again optimized by the genetic algorithm (Tables 3 and 4), this gives additional optimization. In this case joint optimization of both the balancer valves of the risers and the valves of the heaters gives the best result. This effect confirms the theoretical proposition that coordinates search method and gradient methods do not guarantee the achievement of global extremum $[11,26,27]$.

The various advantages and disadvantages of the genetic algorithm and the coordinates search method are illustrated very well by Figure 3, which shows that coordinates search algorithm works faster, but the genetic algorithm allows achieving greater depth of optimization due to the gradual approximation, although it works slower.

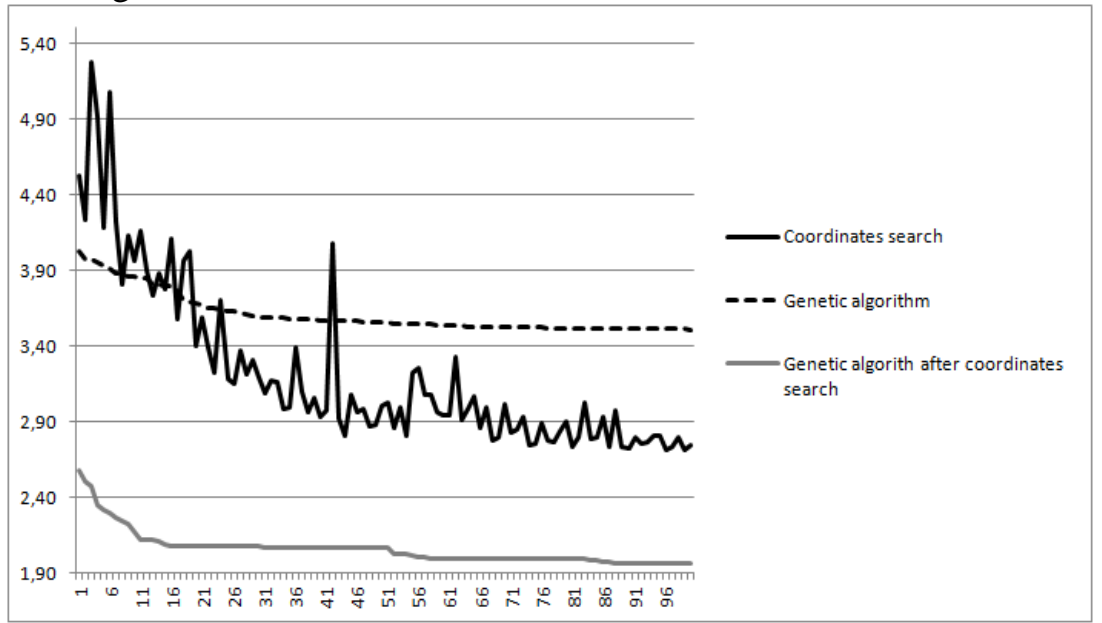

Figure 3. Comparison of optimization history using the coordinates search method and the genetic algorithm, as well as using the genetic algorithm as an additional optimization of coordinates search.

\section{Conclusion}

As the experimental results show, the best result of optimizing the temperature disbalance can be achieved by using joint adjusting of the risers balancing valves and heater valves, if you first apply the optimization of the coordinates search methods and then further optimize it by the genetic algorithm. This fact also confirms the hypothesis put forward in [11]: "Combining a genetic algorithm and gradient methods will allow you to create a more efficient optimization algorithm (than gradient methods) with an acceptable computation time."

\section{References}

[1] Vologdin S V, Jakimovich B A 2015 Methods and algorithms for improving the energy efficiency of a multi-level district heating system: a monograph (Izhevsk: Publishing ISTU named after M.T. Kalashnikov) p 264

[2] Smart City URL: http://city-smart.ru (18.11.2018)

[3] Zvonareva U N, Vankov U V 2015 Energy saving in heat supply systems of large municipal unions, powered from several heat sources News of the Tomsk Polytechnic University. Georesource Engineering 326(11) 75-82

[4] Strizhak P A, Morozov M N 2015 Mathematical modeling of the thermal mode of a building, taking into account the insolation heat gains Bulletin of Tomsk Polytechnic University. Engineering of Georesources 326(8) 36-46

[5] Zakharova A S, Orlova A K 2018 "Smart Home" technology as an energy-saving technology of the future Economy and Entrepreneurship 5(94) 1166-1169 
[6] Lapaeva O F 2010 Transformation of the energy sector of the economy in the transition to energy-saving technologies and renewable energy sources Orenburg State University Bulletin 13(119) 50-56

[7] Shchegolikhina M S 2015 Energy saving technologies are technologies of the future Progressive technologies and processes. Collection of scientific articles of the 2nd International Youth Scientific and Practical Conference in 3 volumes 193-194

[8] Levinson S V 2015 What is new in energy-saving technologies International Journal of Experimental Education 78-80

[9] Balabanov M S, Baboshkina S V and Khamitov R N 2015 Environmental aspects in energysaving policy at the stage of creating intelligent energy systems in Russia with an activeadaptive network Izvestia Tomsk Polytechnic University. Engineering of Georesources 326(11) 141-152

[10] Shuravin A P 2018 Review of optimization problems of thermo-hydraulic modes of buildings Collection of materials of the All-Russian scientific-practical conference with international participation (Stavropol: Center for Scientific Knowledge "Logos") 51-55

[11] Shuravin A P, Vologdin S V 2018 The use of a genetic algorithm to optimize the temperature mode of indoor areas by adjusting the balance valves of the risers Intellectual systems in production 16(2) 113-120

[12] Novitsky N N, Lutsenko A V 2016 Study of the issues and methods of multicriteria optimization of hydraulic modes of distribution heat networks Scientific Herald of the NSTU 3(64) 131-145

[13] Vologdin S V, Moshkin A V 2011 Mathematical model for optimizing the cost of production and transportation of thermal energy in a centralized heating system, in the presence of a pressure and resistance regulator The world of scientific discoveries 8 281-290

[14] Vologdin S V 2011 Mathematical model for optimizing heat flows between buildings of a multi-loop heat network in order to reduce the disbalance of the heat supply system by regulating the nozzles of the elevator nodes The world of scientific discoveries 12 194-204

[15] Basalaev A A, SHnajder D A 2017 The method of optimizing the temperature of the coolant in the system of centralized heat supply of buildings based on simulation Bulletin of the South Ural State University. Series: computer technology, management, electronics 17(1) $15-22$

[16] Manusov V Z, Matrenin P V and Kirgizov A K 2017 Optimization of the distribution of compensating devices in power supply systems based on swarm intelligence Energy Security and Energy Saving 3 28-32

[17] Bajbakov S A, Filatov K V 2012 Optimization of heating networks for the cost of transporting the coolant (optimization of heat transportation) Energetic 12 26-33

[18] Stennikov V A, Hemerzov C A 2018 Application of tree search algorithm and annealing imitation method for circuit-structural optimization of heat networks Software products and systems 2 387-395

[19] Sacaan R, Rudnick H, Lagos T, Ordóñez F, Navarro-Espinosa A and Moreno R 2017 IEEE Manchester PowerTech (Manchester) 1-6

[20] Dolan M J, Davidson E M, Kockar I, Ault G W and McArthur S D J 2012 IEEE Transactions on Power Systems 27 790-799

[21] Kong C, Jovanovic R, Bayram I S and Devetsikiotis M 2017 A hierarchical optimization model for a network of electric vehicle charging stations Energy 10(5)

[22] Wang D, Hu Q, Tang J, Lia H, Li Y, Gao S and Fan M 2017 A kriging model based optimization of active distribution networks considering loss reduction and voltage profile improvement Energy 10(5)

[23] Scardapane S, Panella M and Wang D 2016 A decentralized training algorithm for Echo State Networks in distributed big data applications Neural Networks 78 65-74 
[24] Muñoz E, Márquez-Neila P and Baumela L 2015 Rationalizing Efficient Compositional Image Alignment: The Constant Jacobian Gauss-Newton Optimization Algorithm International Journal of Computer Vision 112(3) 354-372

[25] Chatterjee K, Chmelík M, Gupta R and Kanodia A 2016 Optimal cost almost-sure reachability in POMDPs Artificial Intelligence 234 26-48

[26] Akulich I L 2011 Mathematical programming in examples and problems: Textbook. manual for students of the economy Universities (St. Petersburg: Lan) p 352

[27] Korobeynikov A V 2013 Programming neural networks: a teaching aid for the disciplines "Optimization Methods. Neural Networks","Neurocomputer Systems" and "Fuzzy Logic and Genetic Algorithms" (Izhevsk: Publishing house IzhSTU) p 44

[28] GOST 30494-2011. Residential and public buildings. Indoor microclimate parameters

[29] Rabiner L, Gould B M 1978 Theory and application of digital signal processing (World) p 835 